\title{
Decoherence of Anyonic Charge in Interferometry Measurements
}

\author{
Parsa Bonderson, ${ }^{1}$ Kirill Shtengel, ${ }^{2}$ and J. K. Slingerland ${ }^{3}$ \\ ${ }^{1}$ California Institute of Technology, Pasadena, California 91125, USA \\ ${ }^{2}$ Department of Physics and Astronomy, University of California, Riverside, California 92521, USA \\ ${ }^{3}$ Microsoft Research, Station Q, CNSI Building, University of California, Santa Barbara, California 93106, USA
}

(Received 8 September 2006; published 12 February 2007)

\begin{abstract}
We examine interferometric measurements of the topological charge of (non-Abelian) anyons. The target's topological charge is measured from its effect on the interference of probe particles sent through the interferometer. We find that superpositions of distinct anyonic charges $a$ and $a^{\prime}$ in the target decohere (exponentially in the number of probes particles used) when the probes have nontrivial monodromy with the charges that may be fused with $a$ to give $a^{\prime}$.
\end{abstract}

DOI: 10.1103/PhysRevLett.98.070401

Quantum physics in two spatial dimensions allows for the existence of particles which are neither bosons nor fermions. Instead, the exchange interactions of such "anyons" are described by representations of the braid group [1], which may even be non-Abelian [2]. Recently, there has been a resurgence of interest in anyons, due to increased experimental capabilities in systems believed to harbor them, and also their potential application to topologically protected quantum computation [3]. In this quantum computing scheme, qubits are encoded in nonlocalized, topological charges carried by clusters of nonAbelian anyons. Topological charges decouple from local probes, affording them protection from decoherence. However, this also makes their measurement, which is vital for qubit readout, more difficult, typically requiring interferometry. The most promising candidate system for discovering non-Abelian statistics is the fractional quantum Hall (FQH) state observed at filling fraction $\nu=5 / 2$ [4], which is widely expected to be described by the MooreRead state [5]. Interference experiments, similar to that proposed [6] and only recently implemented [7] for Abelian FQH states, may soon verify the braiding statistics of the $\nu=5 / 2$ state [8]. The analyses in these treatments assume the target particle to be in an eigenstate of topological charge. We show that, when this is not the case, the density matrix of the target particle is diagonalized in the charge basis during the experiment if a simple criterion on the braiding of source and target particles is satisfied: superpositions of distinct anyonic charges $a$ and $a^{\prime}$ decohere as long as the probe particles have nontrivial monodromy with the charge differences between $a$ and $a^{\prime}$, that is, with the charges that fuse with $a$ to give $a^{\prime}$.

We consider a Mach-Zehnder type interferometer (see Fig. 1), though the same methods may be applied to other types, with similar conclusions. A target "particle" A carrying a superposition of anyonic charges [9] is located in the region between the two paths of the interferometer. A beam of probe particles $B_{k}, k=1, \ldots, N$ may be sent into two possible input channels, is passed through a beam splitter $T_{1}$, reflected by mirrors around the central region, passed through a second beam splitter $T_{2}$, and finally
PACS numbers: 03.67.Pp, 03.65.Ta, 03.65.Vf, 05.30.Pr

detected at two possible output channels. The state acquires a phase $e^{i \theta_{\mathrm{I}}}$ or $e^{i \theta_{\mathrm{II}}}$ when a probe particle passes through the bottom or top path around the central region (this may come from background flux, path length differences, phase shifters, etc.) and a separate, independent contribution strictly from the braiding of the probe and target particles, which, for non-Abelian anyons, will be more complicated than a mere phase. If the phases $e^{i \theta_{\mathrm{I}}}$ and $e^{i \theta_{\text {II }}}$ are fixed, or closely monitored, this provides a nondemolitional measurement of the anyonic charge of $A$ [10]. This admittedly idealized setup is similar to one experimentally realized for quantum Hall systems [11], the primary difference being that the number of quasiparticle excitations in the central interferometry region is not fixed in that experiment. While unsuitable for measuring a target charge, this situation may still be used to detect the presence of non-Abelian statistics [12].

The experiment we describe was also considered in the paper [13], where it was referred to as the "many-to-one" experiment. In that paper, the authors use a quantum group inspired approach, where individual particles are assumed to have internal Hilbert spaces, and they study what happens to the internal state of the target particle. In our descriptions of the systems examined, we use the theory of general anyon models (unitary braided tensor categories), which does not ascribe individual particles internal degrees of freedom. Instead, the relevant observables are

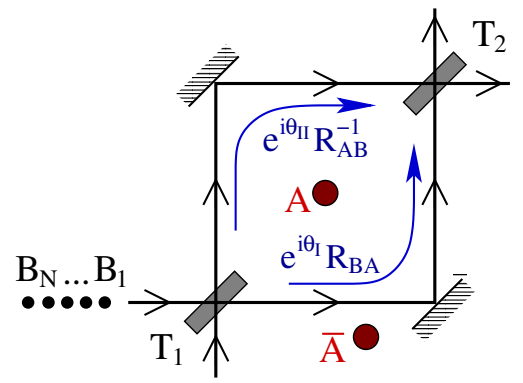

FIG. 1 (color online). A Mach-Zehnder interferometer containing the target anyon(s) $A$, to be probed by the anyons $B_{k}$. (Detectors not shown.) 
the overall anyonic charges of groups of particles (our main result will be stated in terms of the density matrix of an anyon pair $A-\bar{A})$. This is the situation relevant to the topological systems (e.g., FQH states) that we have in mind. We also remove some constraints imposed in [13], specifically, that the probe particles are all identical and have trivial self-braiding.

Let us recall some information about anyon models (see, e.g., [14] for additional details). States in these models may be represented by superpositions of oriented worldline diagrams that give a history of splitting and fusion of particles carrying an anyonic charge. Each allowed fusion/splitting vertex is associated with a (possibly multidimensional) vector space containing normalized bra/ket vectors

$$
\begin{aligned}
& \left(d_{c} / d_{a} d_{b}\right)^{1 / 4} \overbrace{a}^{c} \bigwedge_{b}^{\mu}=\left\langle a, b ; c, \mu l \in V_{a b}^{c}\right. \\
& \left(d_{c} / d_{a} d_{b}\right)^{1 / 4}{ }_{c}^{a} \uparrow^{b}=|a, b ; c, \mu\rangle \in V_{c}^{a b},
\end{aligned}
$$

where $\mu$ labels the basis states of the splitting space $V_{c}^{a b}$ of the charges $a$ and $b$ from charge $c$ and the number $d_{a} \geq 1$ is the quantum dimension of $a$. The factors of $\left(d_{c} / d_{a} d_{b}\right)^{1 / 4}$ are necessary for isotopy invariance, i.e., so the meaning of the diagrams is not changed by continuous deformation. The vacuum is labeled 1 , and has $d_{1}=1$. Since $\operatorname{dim} V_{c}^{a b}=$ 1 when any of $a, b, c$ equals 1 , the basis label is redundant and will be dropped. In fact, the meaning of diagrams is invariant under addition/removal of vacuum lines, so we may drop them and smooth out their vertices. The charge conjugate, or antiparticle, of $a$ is denoted $\bar{a}$, and may also be denoted by reversing the arrow on a line labeled by $a$. Diagrams with multiple vertices correspond to tensor products of vertex spaces. Density matrices may be represented by diagrams with the same numbers of lines emerging at the top and bottom (being combinations of kets and bras). Conjugation of states and operators corresponds to reflecting their diagrams through the horizontal plane while reversing orientations [e.g., Eqs. (1) and (2)]. One may diagrammatically trace out a charge that enters and exits a diagram at the same spatial position by connecting the lines at these positions with an arc that does not interfere with the rest of the diagram (giving zero if the charges do not match). This is actually the quantum trace, which equals the ordinary trace with each sector of overall charge $f$ multiplied by $d_{f}$. Here are some important diagrammatic relations:

$$
R_{a b}={ }_{a}, \quad R_{a b}^{\dagger}=R_{a b}^{-1}=
$$

$$
S_{a b}=\frac{1}{D} \bigcirc_{a}, \quad \bigcup_{a}^{b}=\frac{S_{a b}}{S_{1 b}} \uparrow^{b},
$$

where $d_{a}=D S_{1 a}$ is the value of an unknotted loop carrying charge $a$, and $D=\sqrt{\sum_{a} d_{a}^{2}}$ is the total quantum dimension. Another useful quantity, especially for interference experiments [15], is the monodromy matrix element $M_{a b}=\frac{S_{a b} S_{11}}{S_{1 a} S_{1 b}}$. It has the property $\left|M_{a b}\right| \leq 1$, with $M_{a b}=$ 1 corresponding to trivial monodromy, i.e., the state is unchanged by taking the charges $a$ and $b$ all the way around each other.

Using this formalism, it is important to keep track of all particles involved in a process. We invoke the physical assumption that the particles $A$ and all $B_{k}$ are initially unentangled. This means there are no nontrivial charge lines connecting them, and to achieve this, they must each be created separately from vacuum, with their own antiparticles [16]. We write the initial state of the $A-\bar{A}$ system as

$$
\left|\Psi_{0}\right\rangle=\sum_{a} A_{a}|a, \bar{a} ; 1\rangle
$$

and that of each $B_{k}-\bar{B}_{k}$ system as

$$
\left|\varphi_{k}\right\rangle=\sum_{b, s} B_{b, s}^{(k)}|\bar{b}, b ; 1 ; s\rangle
$$

where $s=\longrightarrow, \uparrow$ indicates in which direction the probe particle is traveling. The probes' antiparticles, $\bar{B}_{k}$, will be taken off to the left and do not participate in the interferometry. The location of the target's antiparticle $\bar{A}$ with respect to the interferometer is important and we will let it be located below the central region, as in Fig. 1.

Utilizing the two-component vector notation $\left(\begin{array}{l}1 \\ 0\end{array}\right)=|\rightarrow\rangle$, $\left(\begin{array}{l}1 \\ 0\end{array}\right)=|\uparrow\rangle$, the two beam splitters, which (along with the mirrors) are assumed to be lossless, are represented by the unitary operators $T_{j}=\left[\begin{array}{cc}t_{j} & r_{j}^{*} \\ r_{j} & -t_{j}^{*}\end{array}\right]$, with $\left|t_{j}\right|^{2}+\left|r_{j}\right|^{2}=1$. The evolution operator for passing the probe particle $B_{k}$ through the interferometer is

$$
\begin{gathered}
U_{k}=T_{2} \Sigma_{k} T_{1} \\
\Sigma_{k}=\left[\begin{array}{cc}
0 & e^{i \theta_{\mathrm{II}} R_{A, B_{k}}^{-1}} \\
e^{i \theta_{\mathrm{I}}} R_{B_{k}, A} & 0
\end{array}\right] .
\end{gathered}
$$

Diagrammatically, this takes the form

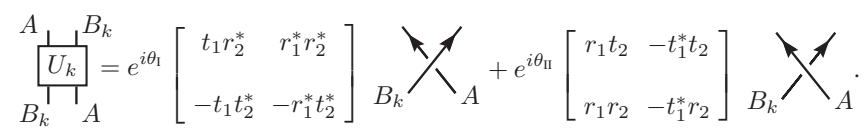

Keeping track of antiparticles, we need $V_{k}=R_{\bar{A}, B_{k}}^{-1}$ for braiding the probe particles with $\bar{A}$, and, adding in each successive $\left|\varphi_{k}\right\rangle$ from the left, we also use the operators 


$$
W_{k}=R_{\bar{B}_{k}, \bar{B}_{k-1}} R_{B_{k}, \bar{B}_{k-1}} \ldots R_{\bar{B}_{k}, \bar{B}_{1}} R_{B_{k}, \bar{B}_{1}}
$$

(and $W_{1}=1$ ), which move the $\bar{B}_{k} B_{k}$ pair from the left to the center of the configuration $\bar{B}_{1} \ldots \bar{B}_{k-1} B_{k-1} \ldots B_{1}$. This may be viewed either as spatial sorting after creation, or, as shown suggestively in Eq. (13), as the temporal condition that each $\bar{B} B$ pair is utilized before creating the next.

The state of the combined system after $N$ probe particles have passed through the interferometer (but have not yet been detected) may now be defined iteratively as

$$
\left|\Psi_{N}\right\rangle=V_{N} U_{N} W_{N}\left|\varphi_{N}\right\rangle \otimes\left|\Psi_{N-1}\right\rangle
$$

Focusing on the $A-\bar{A}$ system, the reduced density matrix, $\rho_{N}^{A}=\operatorname{Tr}_{B^{\otimes N}}\left[\left|\Psi_{N}\right\rangle\left\langle\Psi_{N}\right|\right]$, is obtained by tracing over the $B_{k}$ and $\bar{B}_{k}$ particles. This may be interpreted as ignoring the detection results. Given the placement of $\bar{A}$, one sees that this averaging over detector measurements makes the second beam splitter irrelevant. If we kept track of the measurement outcomes $s_{k}$, we would project with $\left|s_{k}\right\rangle\left\langle s_{k}\right|$ after the $k$ th probe particle. In $\left|\Psi_{N}\right\rangle$, we did not include braiding between the $B_{k}$, but they may be added without changing the results, as they drop out of $\rho_{N}^{A}$ [17].

We will first assume that the probe particles all have the same, definite anyonic charge $b$ and enter through the horizontal leg, so that $\left|\varphi_{k}\right\rangle=|\bar{b}, b ; 1 ; \rightarrow\rangle$ for all $k$, and then later return to the general case. This results in the state

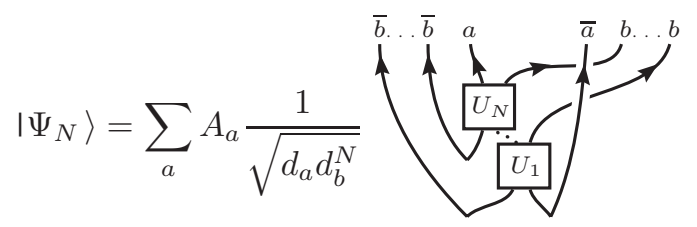

(with directional indices left implicit).

We first consider the case $N=1$. Tracing out the $b$ and $\bar{b}$ lines of $\left|\Psi_{1}\right\rangle\left\langle\Psi_{1}\right|$, and using Eq. (10), one finds that terms cancel to give

$$
\begin{aligned}
& \rho_{1}^{A}=\sum_{a, a^{\prime}} \frac{A_{a} A_{a^{\prime}}^{*}}{\sqrt{d_{a} d_{a^{\prime}}} d_{b}}
\end{aligned}
$$

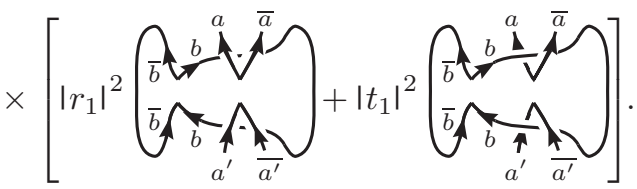

This result simply reflects the fact that all that matters after averaging over measurement outcomes is that the probe particle passes between $A$ and $\bar{A}$ with probability $\left|t_{1}\right|^{2}$, and passes around them with probability $\left|r_{1}\right|^{2}$. Since they are initially unentangled, each additional probe particle has the same analysis as the first, and just results in another loop that passes between $A$ and $\bar{A}$ with probability $\left|t_{1}\right|^{2}$. Noting that an unlinked $b$ loop may be replaced by a factor $d_{b}$, we see that the reduced density matrix for $A$ after passing $N$ probe particles through the interferometer is

$$
\begin{aligned}
\rho_{N}^{A} & =\sum_{a, a^{\prime}} \frac{A_{a} A_{a^{\prime}}^{*}}{\sqrt{d_{a} d_{a^{\prime}}}} \sum_{n=0}^{N}\left(\begin{array}{c}
N \\
n
\end{array}\right)\left|r_{1}\right|^{2(N-n)}\left|t_{1}\right|^{2 n} \\
& =\sum_{a, a^{\prime}} \frac{A_{a} A_{a^{\prime}}^{*}}{\sqrt{d_{a} d_{a^{\prime}}}} \sum_{(e, \alpha, \beta)}\left[\left(F_{a^{\prime}, \overline{a^{\prime}}}^{a, \bar{a}}\right)^{-1}\right]_{1,(e, \alpha, \beta)} \sum_{n=0}^{N}\left(\begin{array}{c}
N \\
n
\end{array}\right)\left|r_{1}\right|^{2(N-n)}\left|t_{1}\right|^{2 n} \\
& \left.=\sum_{a, a^{\prime}} \frac{A_{a} A_{a^{\prime}}^{*}}{\sqrt{d_{a} d_{a^{\prime}}}} \sum_{(e, \alpha, \beta),(f, \mu, \nu)}^{n \times b}\left[\left(F_{a^{\prime}, \bar{a}^{\prime}}^{a, \bar{a}}\right)^{-1}\right]_{1,(e, \alpha, \beta)}{ }_{\left(\left|r_{1}\right|^{2}+\left|t_{1}\right|^{2}\right.} M_{b e}\right)^{N}\left[F_{a^{\prime}, \bar{a} \bar{a}^{\prime}}^{a, \bar{a}}\right]
\end{aligned}
$$

where the relations in Eq. (5) were used to remove all the $b$ loops, allowing us to perform the sum over $n$, before applying $F$ in the last step. The intermediate charge label $e$ represents the difference between the charges $a$ and $a^{\prime}$, taking values that may be fused with $a^{\prime}$ to give $a$ (the $F$ symbols vanish otherwise). Notice the potential for this process to transfer an overall anyonic charge $f$ to the $A-\bar{A}$ system.

From this result we see, noting $\left|t_{1}\right|^{2}+\left|r_{1}\right|^{2}=1$, that taking the limit $N \rightarrow \infty$ will exponentially kill off the $e$ channels with $M_{b e} \neq 1$, and preserve only those which have trivial monodromy with $b, M_{b e}=1$. The interpretation of $M_{b e}=1$ is that $a$ and $a^{\prime}$ have a difference charge $e$ that is invisible (in the sense of monodromy) to the charge $b$, and so the corresponding fusion channel remains unaffected by the probe. In general, the only $e$ channels guaranteed to always survive this process (even for the most general $B_{k}$ states) have trivial monodromy with all charges. This always includes $e=1$ (and for modular theories/TQFTs is the only such charge), which requires that $a=a^{\prime}$. Tracing over the $A$ and $\bar{A}$ particles gives $\operatorname{Tr}\left[\rho_{N}^{A}\right]=1$ as expected, but by considering the intermediate channels, one also finds that the entire contribution to this trace is from $e=1$. We should also note that some terms may alternatively be killed off due to their corresponding $F$ symbols having zero values.

Defining $\rho^{A} \equiv \lim _{N \rightarrow \infty} \rho_{N}^{A}$, and denoting by $e_{b}$ the intermediate charges that have trivial monodromy with $b$, we 
get the final result (converted back into bra/ket notation, with an extra factor of $d_{f}$ inserted for compatibility with the ordinary trace)

$$
\begin{aligned}
\rho^{A}= & \sum_{a, a^{\prime}} A_{a} A_{a^{\prime}}^{*} \sum_{\left(e_{b}, \alpha, \beta\right),(f, \mu, \nu)}\left[\left(F_{a^{\prime}, \overline{a^{\prime}}}^{a, \bar{a}}\right)^{-1}\right]_{1,\left(e_{b}, \alpha, \beta\right)} \\
& \times\left[F_{a^{\prime}, \bar{a}^{\prime}}^{a, \bar{a}}\right]_{\left(e_{b}, \alpha, \beta\right),(f, \mu, \nu)} \sqrt{d_{f}}|a, \bar{a} ; f, \mu\rangle\left\langle a^{\prime}, \overline{a^{\prime}} ; f, \nu\right| .
\end{aligned}
$$

We now return to the case of general probe particle states as given in Eq. (7). Since tracing requires the charge on a line to match up, a similar analysis as before applies. For the result, we simply replace $\left(\left|r_{1}\right|^{2}+\left|t_{1}\right|^{2} M_{b e}\right)^{N}$ in Eq. (15c), with

$$
\prod_{k=1}^{N}\left[1-\sum_{b}\left|B_{b, \rightarrow}^{(k)} t_{1}+B_{b, r}^{(k)} r_{1}^{*}\right|^{2}\left(1-M_{b e}\right)\right] .
$$

This term determines the rate at which the $A$ system decoheres, and will generically vanish exponentially as $N \rightarrow \infty$ unless $e$ has trivial monodromy (in which case this term simply equals 1 ). In some cases, complete decoherence may even be achieved with a single probe step. By setting $\left|r_{1}\right|=0$ and $\left|t_{1}\right|=1$ in Eq. (15c), we may do away with the interferometer and interpret the result as decoherence from stray anyons passing between $A$ and $\bar{A}$, which is important to consider as a source of errors in a quantum computation.

As a practical example, we apply the results to the Ising anyon model, which captures the essence of the MooreRead state's non-Abelian statistics. For the initial state $\left|\Psi_{0}\right\rangle=A_{1}|1,1 ; 1\rangle+A_{\psi}|\psi, \psi ; 1\rangle+A_{\sigma}|\sigma, \sigma ; 1\rangle$, using $\sigma$ probes (which have trivial monodromy only with 1 ) gives

$$
\begin{aligned}
\rho^{A}= & \left|A_{1}\right|^{2}|1,1 ; 1\rangle\left\langle 1,1 ;\left.1|+| A_{\psi}\right|^{2} \mid \psi, \psi ; 1\right\rangle\langle\psi, \psi ; 1| \\
& +\left|A_{\sigma}\right|^{2} \frac{1}{2}(|\sigma, \sigma ; 1\rangle\langle\sigma, \sigma ; 1|+| \sigma, \sigma ; \psi\rangle\langle\sigma, \sigma ; \psi|),
\end{aligned}
$$

which exhibits loss of all coherence. For $\psi$ probes (which have trivial monodromy with both 1 and $\psi$ ) the result

$$
\begin{aligned}
\rho^{A}= & \left|A_{1}\right|^{2}|1,1 ; 1\rangle\left\langle 1,1 ; 1\left|+A_{\psi} A_{1}^{*}\right| \psi, \psi ; 1\right\rangle\langle 1,1 ; 1| \\
& +A_{1} A_{\psi}^{*}|1,1 ; 1\rangle\left\langle\psi, \psi ;\left.1|+| A_{\psi}\right|^{2} \mid \psi, \psi ; 1\right\rangle\langle\psi, \psi ; 1| \\
& +\left|A_{\sigma}\right|^{2}|\sigma, \sigma ; 1\rangle\langle\sigma, \sigma ; 1|
\end{aligned}
$$

shows decoherence only between $\sigma$ and the other charges.

The decoherence effect described in this Letter is due to measurements being made by probe particles. Keeping track of these measurement outcomes, one generically finds collapse of the target system state into subspaces where the difference charge has trivial monodromy with the probes [18]. If this includes only the $e=1$ subspaces, the target collapses onto a state of definite charge. One may also consider completely general initial $A$ and $B_{k}$ systems described by density matrices, but as long as they are all still unentangled, the resulting behavior is qualitatively similar. It may also be physically relevant in some cases to allow initial entanglement between the probes, though this greatly complicates the analysis and results. These generalizations will be addressed in [18].

We thank A. Kitaev, I. Klich, and especially J. Preskill for illuminating discussions, and the organizers and participants of the KITP Workshop on Topological Phases and Quantum Computation where this work was initiated. We would also like to acknowledge the hospitality of the IQI, the KITP, and Microsoft Project Q. This work was supported in part by the NSF under Grants No. PHY-0456720 and No. PHY99-07949, and the NSA under ARO Contract No. W911NF-05-1-0294.

[1] J. M. Leinaas and J. Myrheim, Nuovo Cimento B 37, 1 (1977); F. Wilczek, Phys. Rev. Lett. 48, 1144 (1982); 49, 957 (1982).

[2] G. A. Goldin, R. Menikoff, and D. H. Sharp, Phys. Rev. Lett. 54, 603 (1985); J. Fröhlich and F. Gabbiani, Rev. Math. Phys. 2, 251 (1990).

[3] A. Y. Kitaev, Ann. Phys. (N.Y.) 303, 2 (2003); J. Preskill, in Introduction to Quantum Computation, edited by H.-K. Lo, S. Popescu, and T.P. Spiller (World Scientific, Singapore, 1998); M.H. Freedman, M.J. Larsen, and Z. Wang, Commun. Math. Phys. 227, 605 (2002).

[4] R. Willett et al., Phys. Rev. Lett. 59, 1776 (1987); W. Pan et al., Phys. Rev. Lett. 83, 3530 (1999).

[5] G. Moore and N. Read, Nucl. Phys. B360, 362 (1991); C. Nayak and F. Wilczek, Nucl. Phys. B479, 529 (1996).

[6] C. de C. Chamon et al., Phys. Rev. B 55, 2331 (1997).

[7] F.E. Camino, W. Zhou, and V. J. Goldman, Phys. Rev. B 72, 075342 (2005); Phys. Rev. Lett. 95, 246802 (2005).

[8] E. Fradkin et al., Nucl. Phys. B516, 704 (1998); S. Das Sarma, M. Freedman, and C. Nayak, Phys. Rev. Lett. 94, 166802 (2005); A. Stern and B. I. Halperin, Phys. Rev. Lett. 96, 016802 (2006); P. Bonderson, A. Kitaev, and K. Shtengel, Phys. Rev. Lett. 96, 016803 (2006).

[9] Since localized charges cannot be superimposed, when we refer to "a particle having a superposition of charges," we really mean several (quasi-)particles, treated collectively.

[10] Decoherence in the charge basis is actually independent of these phases, as they drop out of the density matrix for $A$.

[11] Y. Ji et al., Nature (London) 422, 415 (2003).

[12] D. E. Feldman and A. Kitaev, cond-mat/0607541.

[13] B. J. Overbosch and F. A. Bais, Phys. Rev. A 64, 062107 (2001).

[14] J. Preskill (2004), lecture notes, URL http://www. theory.caltech.edu/ preskill/ph219/topological.ps; A. Kitaev, Ann. Phys. (N.Y.) 321, 2 (2006).

[15] P. Bonderson, K. Shtengel, and J.K. Slingerland, Phys. Rev. Lett. 97, 016401 (2006).

[16] These "particle-antiparticle pairs" may really be multiple pair-created particles that are made to interact amongst each other as needed and then split into two groups that are henceforth treated collectively.

[17] Superpositions of braiding may, however, change these results.

[18] P. Bonderson, K. Shtengel, and J. K. Slingerland (to be published). 